\title{
EBL signature on VHE gamma photons
}

\author{
Daniel Mazin ${ }^{* \dagger}$ \\ MPI for Physics, Munich, Germany \\ now at: ICRR, University of Tokyo, Japan \\ E-mail: mazin@mpp.mpg.de
}

\begin{abstract}
Very high energy (VHE, E > $30 \mathrm{GeV}$ ) gamma-rays are absorbed via interaction with low-energy photons from the extragalactic background light (EBL) if the involved photon energies are above the threshold for electron-positron pair creation. The VHE gamma-ray absorption, which is energy dependent and increases strongly with redshift, distorts the VHE energy spectra observed from distant objects. The observed energy spectra of the AGNs carry, therefore, an imprint of the EBL. The detection of hard VHE gamma-ray spectra of distant sources $(z=0.11-0.60)$ by H.E.S.S., MAGIC and VERITAS enabled to set strong upper limits on the EBL density, using certain basic assumptions about blazar physics. Recently, Fermi/LAT and H.E.S.S. data led to first clear detection of the EBL. Here, we critically review the assumptions for the constraints and discuss perspectives for the EBL and cosmology studies with the next generation observatory for ground-based gamma-ray observation CTA.
\end{abstract}

Science with the New Generation of High Energy Gamma-ray experiments, 10th Workshop - Scineghe2014 04-06 June 2014

Lisbon - Portugal

\footnotetext{
* Speaker.

$\dagger$ Thanks to Dmitri Semikoz (APC, Paris) and Andreas Zech (Observatoire de Paris) for fruitful discussions, as well as Catherine Boisson, Helene Sol and Andreas Zech (LUTH, Observatoire de Paris) for hosting me in Paris
} 


\section{Extragalactic Background Light}

During the star and galaxy formation history a diffuse extragalactic radiation field has been accumulated in the ultraviolet to far infrared wavelength regimes. This radiation field, commonly referred to as the extragalactic background light (EBL), is the second largest, in terms of the contained energy, background after the Cosmic Microwave Background of $2.7 \mathrm{~K}$ (CMB). While the CMB conserves the structure of the universe at the moment of the decoupling of matter and radiation following the Big Bang (at redshift $\mathrm{z} \approx 1000$ ), the EBL is a calorimetric measure of the entire radiant energy released by processes of structure formation that have occurred since the decoupling.

A closer look to the UV - infrared backgrounds is given in Figure 1, left plot. From right to left, the spectral energy distributions of the three major components are shown: the cosmic microwave background (CMB), the cosmic infrared background (CIB), and the cosmic optical background (COB). The COB, peaking at around $1 \mu \mathrm{m}$ is believed to originate directly from stars. The CIB, having its peak at $\sim 100 \mu \mathrm{m}$, results mostly from starlight that has been absorbed by dust inside galaxies and reemitted at higher wavelengths. Throughout this paper, we will refer to COB and CIB together as EBL. Other contributions, like emission from AGN and quasars are expected to produce no more than 5 to $20 \%$ of the total EBL density in the mid IR (see e.g. [1] and references therein).
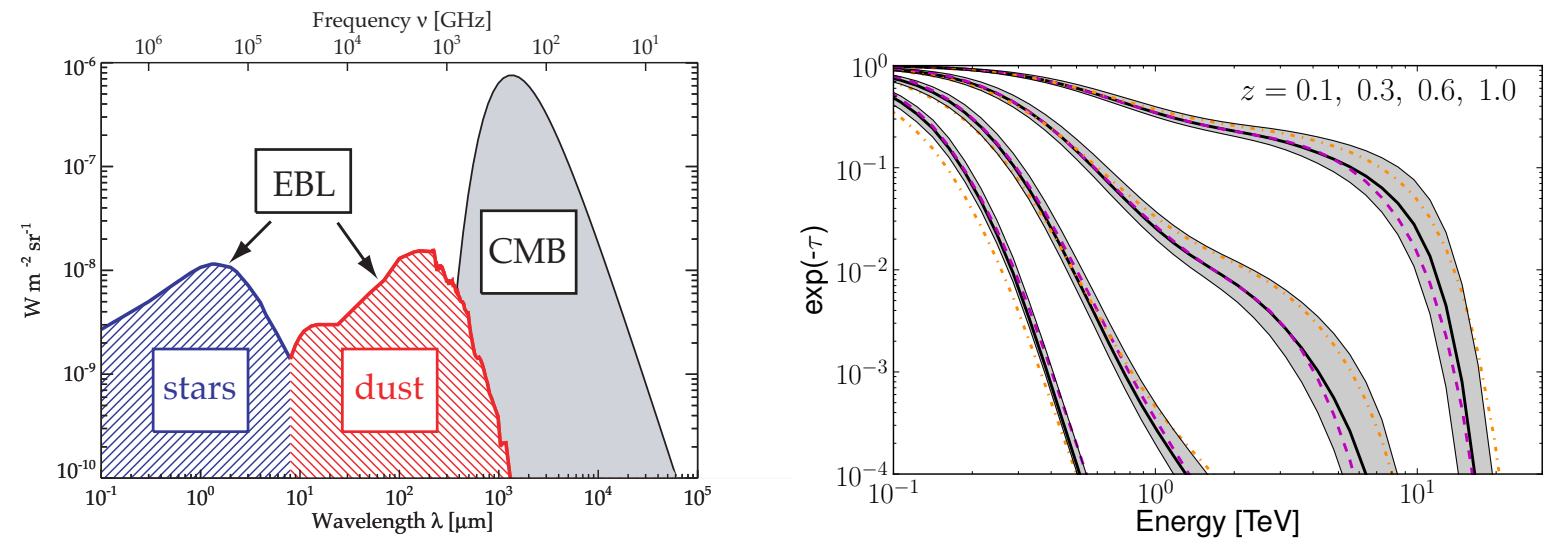

Figure 1: Left: Schematic Spectral Energy Distributions (SED) of the most intense backgrounds in the universe. From right to left: the cosmic microwave background (CMB), the cosmic infrared background (CIB) and the cosmic optical background (COB). The last two components together are called EBL. Plot adopted from [2]. Right: Attenuation coefficients $\exp (-\tau)$ as a function of $\gamma$-ray energy. The model and uncertainties are from [7]. The curves represent the expected attenuation of the VHE $\gamma$-ray spectra for different source distances.

The EBL is difficult to measure directly due to strong foregrounds from our solar system and the Galaxy. The observation of distant sources of VHE $\gamma$-rays using Imaging Air Cherenkov Telescopes (IACT, such as H.E.S.S., MAGIC or VERITAS) provides a unique indirect measurement of the EBL. The precision of the EBL constraints set by the IACT improved remarkably in the last few years. In addition to the IACTs, the LAT instrument on board the Fermi satellite operating in the energy regime between $100 \mathrm{MeV}$ and $>300 \mathrm{GeV}$ has shown a high sensitivity to the EBL imprint on the energy spectra of distant sources. Even if the event statistics is rather limited for 
high energy $(\mathrm{E}>30 \mathrm{GeV})$ events of the $\sim 1 \mathrm{~m}^{2}$ instrument, the combination of the event statistics at lower energies, in the EBL unabsorbed regime, and the large number of detected sources help to significantly constrain the EBL density. Contemporaneously with the IACT and Fermi/LAT constraints, there has been rapid progress in resolving a significant fraction of this background with the deep galaxy counts at infrared wavelengths from the Infrared Space Observatory (ISO) and from the Spitzer satellite as well as at sub-millimeter wavelengths from the Submillimeter Common User Bolometer Array (SCUBA) instrument. The current status of direct and indirect EBL measurements (excluding limits from the IACTs) can be found in [3].

In total, the collective limits on the EBL between the UV and far-IR confirm the expected two peak structure, although the absolute level of the EBL density remains uncertain by a factor of 2 to 10. In addition to this consistent picture, several direct measurements in the near IR have also been reported (e.g. [4]), significantly exceeding the expectations from source counts (see [5] and [6] for recent reviews). If this claimed excess of the EBL is real, it might be attributed to emissions by the first stars in the history of the universe. These so-called Population III stars are believed to be heavy stars with a very low metallicity.

\section{VHE $\gamma$-rays from blazars as a probe of the EBL}

On the way from the source to the observer, VHE $\gamma$-rays can suffer absorption losses by interaction with the low energy photons of the EBL. The corresponding reaction is the pair-production of an electron-positron pair:

$$
\gamma_{\mathrm{VHE}}+\gamma_{\mathrm{EBL}} \longrightarrow e^{+}+e^{-} \text {with } E_{\gamma_{\mathrm{VHE}}} \cdot E_{\gamma_{\mathrm{EBL}}}>\left(m_{e} c^{2}\right)^{2}
$$

The optical depth of the VHE $\gamma$-rays, $\tau(E)$, emitted at the redshift $z$, can then be calculated by solving the three-fold integral:

$$
\begin{aligned}
\tau\left(E_{\gamma}, z\right) & =\int_{0}^{z} \mathrm{~d} \ell\left(\mathrm{z}^{\prime}\right) \int_{-1}^{1} d \mu \frac{1-\mu}{2} \int_{\varepsilon_{t h}^{\prime}}^{\infty} d \varepsilon^{\prime} n\left(\varepsilon^{\prime}, z^{\prime}\right) \sigma_{\gamma \gamma}\left(\varepsilon^{\prime}, E^{\prime}, \mu\right) \\
E_{\gamma} & :=\text { gamma-ray energy in the observer frame work } \\
E^{\prime} & :=\text { redshifted gamma-ray energy at the place of pair creation } \\
\varepsilon^{\prime} & :=\text { redshifted energy of soft photons at the place of pair creation } \\
\mu & :=\cos \theta \\
n(\varepsilon) & :=\text { EBL energy density } \\
\mathrm{d} \ell(\mathrm{z}) & :=\text { distance element }
\end{aligned}
$$

The expected optical depth for VHE $\gamma$-ray fluxes from sources at different redshifts is shown in Fig. 1, right panel. Hereby, the model from [7] is used to estimate the EBL density. One can see that the optical depth is not only redshift but also energy dependent. It is evident that a low threshold of a VHE $\gamma$-ray detector is essential to observe distant sources.

There are two major aspects concerning the interconnection between VHE $\gamma$-rays and the EBL:

1. The EBL leaves a unique imprint on the VHE spectra. This imprint can be used to study the EBL. 
2. The impact of EBL onto VHE spectra is fundamental. We need to understand EBL in order to study intrinsic properties of the VHE $\gamma$-ray sources.

Concerning point (1): From a single observed energy spectrum of a distant VHE $\gamma$-ray source, it is rather difficult if not impossible to distinguish between the imprint of the EBL and intrinsic features of the source. Observed features can be source inherent due to an internal absorption inside the source or due to a source, which does not provide necessary conditions for acceleration of charged particles to high enough energy. A way to pin-point the EBL impact is to use population studies of many extragalactic sources: whereas the intrinsic features might be different, the imprint of the EBL is the same (at a given redshift). With the current population of VHE $\gamma$-ray sources, it is only possible to set limits on the EBL by arguing that the observed spectra contain at least the imprint of the EBL and by making some assumptions on the intrinsic source spectra.

Note that only extragalactic VHE $\gamma$-ray emitters suffer from the absorption by the EBL. For the galactic sources, the effect is negligible up to energies of about $100 \mathrm{TeV}$. For higher energies, the absorption by the photon field of the CMB starts to be important.

The observed VHE $\gamma$-ray spectrum of a source $\left(F_{\text {obs }}\right)$ can be used to reconstruct the intrinsic spectrum $\left(F_{\text {intr }}\right)$, i.e. the one at the source location:

$$
F_{\text {intr }}=\exp (\tau) \times F_{\text {obs }},
$$

whereas $\tau$ is an energy and distance dependent optical depth of VHE $\gamma$-rays. By measuring the observed spectrum of a source and inferring certain limits on the intrinsic spectrum of the source, it is thus possible to constrain $\tau$ and, therefore, to constrain the EBL density.

Flat Spectrum Radio Quasars (FSRQ) and BL Lacs are subclasses of AGNs, which have their jet aligned close to the line of sight of the observer. The two subclasses are jointly called "blazars". According to an accepted paradigm, in AGNs VHE $\gamma$-ray photons are produced by VHE electrons or protons, which are accelerated to VHE energies through a shock acceleration. Lorentz factors in the jets and the jet alignment towards the observer enhance chances to detect VHE $\gamma$-ray emission for blazars. Blazars are indeed the so far most prominent class of VHE $\gamma$-ray emitting extragalactic objects. In the shock acceleration models, the hardest index obtained for the accelerated particles is $s=1.5$ (see e.g. [8]). In the case of protons interacting with ambient plasma, the resulting $\gamma$-ray spectrum has the same slope as $s$, i.e. $\Gamma_{\text {int }}=1.5$. In the case of electrons, the spectrum of the $\gamma$-rays emitted through inverse Compton scattering is expected to be steeper than 1.5 under most circumstances. Therefore, different authors $[9,10,11]$ assumed $\Gamma_{\text {int }}=1.5$ to be the hardest possible intrinsic spectrum, and using this criterion stringent EBL limits were derived. It was, however, argued that stochastic electron acceleration [12], truncated electron spectra [13] or internal absorption (e.g. $[14,15])$ can lead to even harder VHE $\gamma$-ray spectra than with an index of $\Gamma_{\text {int }}=1.5$ although up to now no harder spectra with $\Gamma_{\text {int }}<1.5$ have been observed at lower energies, where no EBL absorption takes place.

\section{The status and the prospects of the indirect EBL constraints}

Several constraints on the EBL were set in the recent years using data from the IACTs and Fermi/LAT (e.g. $[9,10,11])$. The first constraints were made using individual sources only, which 
led to criticism that the limits are source specific and if the selected sources are some what "special" the derived EBL limits would be model-dependent because the underlying assumption for the constraints is that the VHE sources are "typical".

In order to avoid this dependency [16] performed a scan over many hypothetical EBL realizations (over 8 million different ones). The authors also tested all available blazar spectra (until 2006) to generalize the EBL limits. The derived limits favor a low EBL level and are in good agreement with galaxy counts from the optical to the mid infrared regimes. Again, the cosmological origin of the near infrared excess (e.g.[4]) can be ruled out even for the extreme case of $\Gamma_{\text {int }}=2 / 3$. The study was updated in 2012 by [3] with more sources confirming the findings from the earlier work, see Figure 2.

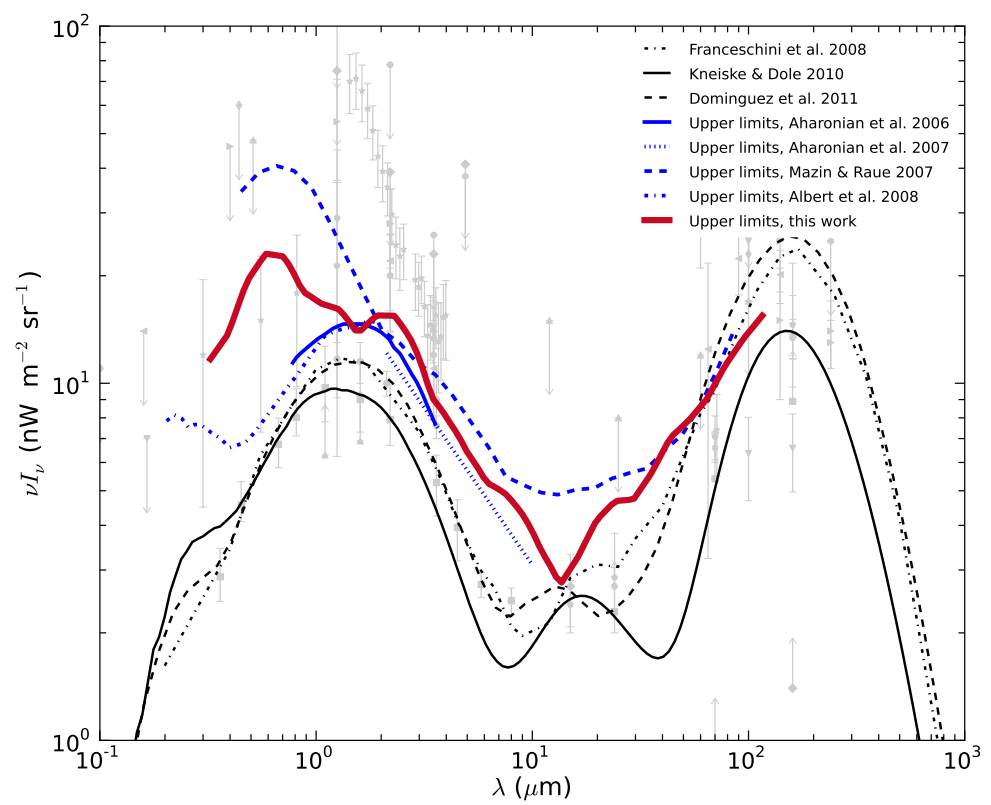

Figure 2: Upper limits on the EBL density as derived in [3].

More recently, in independent works by [17] and [18], it was argued that available data from the detected VHE blazars are already sufficient to measure the EBL, not just derive upper limits on the EBL density. The results, however, either assume some specific AGN model to be at work [18] or a power law shape of the intrinsic photon spectra at energies between $\mathrm{GeV}$ and $\mathrm{TeV}$ regimes [17].

The Fermi/LAT collaboration also derived limits on the EBL density using two years of LAT data [19]. With more data, the LAT collaboration succeeded to derive EBL density using an assumption of the smoothness of the blazar spectra and arguing that the lever arm at low energies (where no EBL absorption is taking place) is a very good proxy of the intrinsic spectrum in the EBL-affected energy regime [20]. A clear advantage of the technique is the usage of the global spectral likelihood fit, leaving the normalization of the EBL as the only free parameter. The derived EBL density is, within some 20-50\% statistical uncertainty on the level of the light from resolved galaxies. This study shows also the first results of the EBL evolution, i.e. how the EBL 
evolved in time, which is an important test of the galaxy and star formation models.

In a parallel independent work by the H.E.S.S. collaboration, similar study was done using high energy photons from blazars, that were measured with the H.E.S.S IACTs [21]. Even if the farthest source used in this study was at $\mathrm{z}=0.2$, the result also ruled out a no-EBL scenario on a level of more than 5 standard deviations.

A summary of the current limits on the EBL density is shown in Figure 3. The X-axis denotes the redshift and the Y-axis the measured EBL density normalized to the density predicted in the model by [22], which is in a good agreement with the models by, e.g., [23, 7, 24]. Typical error of the measurement is $20-50 \%$, which is not sufficiently small to distinguish between the individual EBL models. However, it is an important result showing that the technique works fine and once more blazar spectra with higher precision will be measured, the EBL measurement will also improve.

Cherenkov Telescope Array (CTA) is the next generation IACT array, with ten-times better sensitivity than the current IACTs and an extended energy coverage between $30 \mathrm{GeV}$ and $300 \mathrm{TeV}$ [25]. The telescopes are now under design and in the prototyping phase with an aim to start scientific observations in both hemispheres by 2020. Several studies were performed to investigate the power of CTA to the cosmology and EBL, see, e.g., [26, 27]. The power to resolve the EBL density with CTA, assuming there are enough sources of high energy photons, is illustrated in Figure 3 by the red solid line. This particular simulation assumes measurement of 10 blazars per redshift bin for $10 \mathrm{~h}$ each source and an averaged source flux of about $50 \%$ the one of the Crab nebula at $100 \mathrm{GeV}$. Though this may sound like a very high flux from too many sources, the time scale when such flares need to be accumulated is long: 20 years. In the last 10 years of observations with IACTs, about 5-10 blazar flares on that flux level were measured per year. Therefore, the assumption is realistic. The statistical limits from such EBL measurement can become even tighter if the sources will be found at a higher flux level increasing the photon statistics in the EBL affected regime. However, systematic uncertainties will dominate the measurement when trying to resolve the EBL at a level better than 5-10\% uncertainty. Still, CTA promises to become a great instrument to study EBL density and EBL evolution.

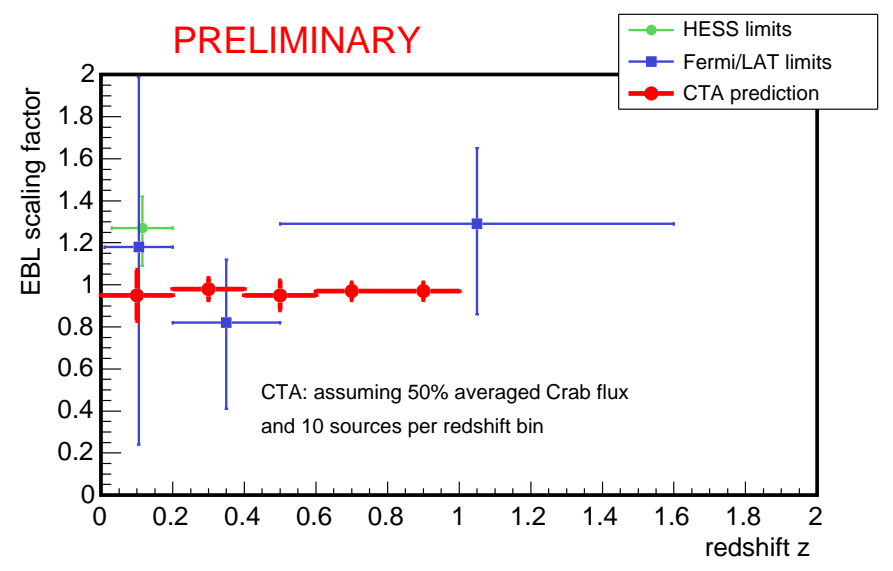

Figure 3: First steps towards resolving the EBL evolution. H.E.S.S. and Fermi/LAT results are shown as well as a prediction for CTA. 


\section{Pile-ups in the energy spectra of VHE sources}

In the same time with the EBL constraints and measurements derived using VHE spectra of blazars, there is an increasing concern that many de-absorbed (i.e. corrected for the EBL absorption) spectra show a pile-up at highest energies measured, in the range $300 \mathrm{GeV}$ to tens of $\mathrm{TeV}$, depending on the source. The pile-up effect in the de-absorbed spectra was measured by [29] with a combined significance of 4.2 standard deviations. Also, in a recent publication by the VERITAS collaboration [30] the de-absorbed spectrum of PKS 1424+240 showed a possible pile up even if assuming the lower limit on the redshift of the source $(\mathrm{z}=0.6)$, which is uncertain (see Figure 4). One of the explanations of the pile-up effect, if real, is the line-of-sight interactions of high-energy cosmic rays with cosmic microwave background radiation (CMBR) and EBL. In this scenario the secondary gamma rays are emitted relatively close to the observer, which provides a plausible explanation of the observed hard spectra, see e.g. [31, 32,33]. Some other possibilities also exist, including Lorentz variance violation or invoking axion-like new particles that oscillate into gamma rays, e.g. [28, 34], preventing strong absorption of the gamma-ray flux by the EBL. However, there is no solid, beyond systematic uncertainties, evidence yet that the pile-up effect is real and not some combination effect of spectral energy reconstruction in some sources and uncertainties in redshift determination.

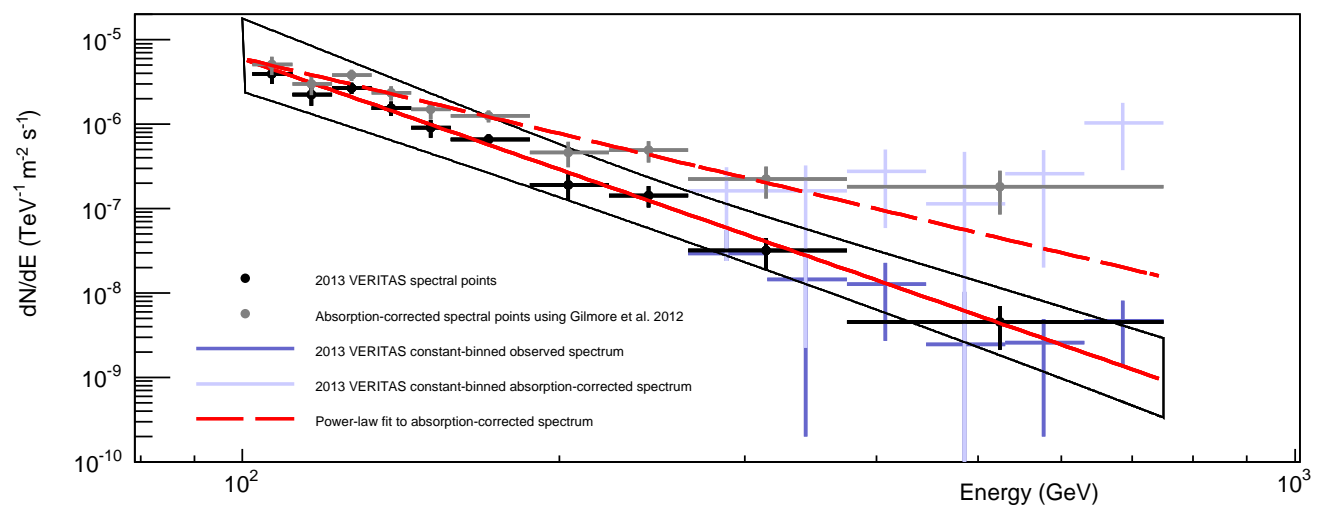

Figure 4: The VHE spectrum of PKS 1424+240 derived from the 2013 VERITAS dataset. The solid red line represents the power-law fit to the observed data. These data are shown with an envelope representing a $40 \%$ systematic error on the source flux and index error of \pm 0.3 . The data are also shown after correction for EBL-absorption by [24], assuming $z=0.6035$ (gray points), along with the power-law (long-dashed line) fit to the absorption-corrected data. Figure from [30].

\section{Fermi/LAT sources for EBL and cosmology studies with CTA}

Finally, we investigate if there will be a sufficient amount of blazars that can be used by CTA to constrain and measure the EBL. In a study, that we are going to publish elsewhere, we extrapolate the Fermi/LAT measured sources into the CTA domain and simulate CTA response to those after 10-20 h of observation of each source. Minimal zenith angle of observation as well as the EBL attenuation are taken into account. Two CTA observatories, one in the North and one in the South, are assumed. The input of the study is the Fermi/LAT hard source catalog, 1FHL 
[35]. Using only sources from this catalog alone, and assuming averaged flux state, we obtain a catalog of potential CTA blazars, see Figure 5. The Figure shows blazars in Galactic coordinates. The black dots depict the blazars from 1FHL that have measured photons with $E>50 \mathrm{GeV}$, the red dots potential CTA blazars and in blue the already detected IACT sources among the 1FHL catalog. As one can see, many of the already detected IACT blazars fall below the CTA 10-h sensitivity. This is typically because of two reasons: a) (rare) the power law extrapolation from 1FHL is too pessimistic or b) (common) the sources were measured with IACTs during gammaray flaring activity. We, therefore, conclude, that the study provides a low limit on the sources that will be detected with CTA. Redshift dependent numbers of CTA blazars are shown in Table 1. It is clear that to study distant universe, here at $z>0.4$, CTA needs to catch sources during gamma-ray flaring activity. In order to maximize the chances to catch blazars during flare activity, an extensive effort of source monitoring must be performed at several wavelength, including the gamma-ray band.

As the number of distant $(\mathrm{z}>0.4)$ sources to be seen with CTA is in order of 25 it is important to re-observe detected sources, mainly if observed in flaring states. Whereas the emission region and flare characteristics might be different at every flare, the EBL imprint on the energy spectrum will remain the same, re-detecting which would make the EBL measurements more solid.

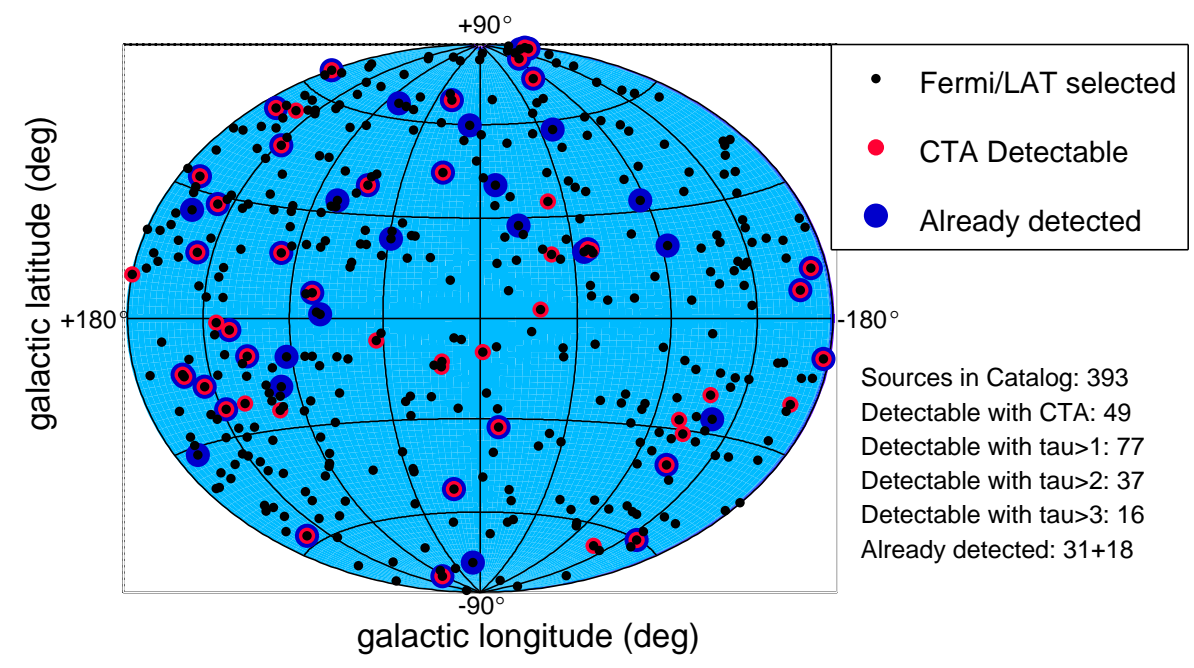

Figure 5: Sky map in galactic coordinates with potential and already detected sources for EBL and cosmology studies. 1FHL blazars that have photons with $E>50 \mathrm{GeV}$ are shown in black dots, already detected blazars among them are blue dots, and potentially detectable blazars with CTA assuming averaged flux state are in red.

\section{References}

[1] I. Matute, F. La Franca, F. Pozzi, et al., Astronomy \& Astrophysics 451, 443-456 (2006).

[2] H. Dole, G. Lagache, Puget, et al., Astronomy \& Astrophysics 451, 417-429 (2006).

[3] M. Meyer, M. Raue, D. Mazin and D. Horns, Astronomy \& Astrophysics, 542, A59 (2012). 


\begin{tabular}{l|c|c|c|c|c||r}
\hline & $z<0.2$ & $0.2<z<0.4$ & $0.4<z<0.6$ & $0.6<z<0.8$ & $z>0.8$ & Total \\
\hline South & 19 & 17 & 4 & 1 & 3 & 63 \\
North & 33 & 16 & 12 & 3 & 2 & 76 \\
\hline South+North & 52 & 33 & 16 & 4 & 5 & 139 \\
\hline
\end{tabular}

Table 1: Prediction for minimum number of blazars that will be detected with CTA for an exposure of 10-20 $\mathrm{h}$ each. The prediction is preliminary and is based on Fermi/LAT 1FHL catalog alone.

[4] T. Matsumoto, S. Matsuura, H. Murakami, M. Tanaka, M. Freund, M. Lim, M. Cohen, M. Kawada \& M. Noda, The Astrophysical Journal 626, 31-43 (2005), astro-ph / 0411593.

[5] M. G. Hauser \& E. Dwek, Annual Review of Astronomy \& Astrophysics 39, 249 (2001).

[6] A. Kashlinsky, Phys. Rep. 409, 361-438 (2005), astro-ph / 0412235.

[7] A. Dominguez, J. Primack, D. J. Rosario, et al., MNRAS 410, 2556-2578 (2011).

[8] M. A. Malkov \& L. O’C Drury, Reports on Progress in Physics 64, 429-481 (2001).

[9] F. A. Aharonian, A. G. Akhperjanian, M. Beilicke, et al., Astronomy \& Astrophysics 403, 523 (2003), astro-ph/0301437.

[10] F. Aharonian, A. G. Akhperjanian, A. R. Bazer-Bachi, et al., Nature 440, 1018-1021 (2006).

[11] J. Albert, E. Aliu, H. Anderhub, et al., Science 320, 1752- (2008), arXiv: 0807.2822.

[12] F. W. Stecker, M. G. Baring \& E. J. Summerlin, The Astrophysical Journal, Letters 667, L29-L32 (2007), arXiv:0707.4676.

[13] K. Katarzyński, G. Ghisellini, F. Tavecchio, J. Gracia \& L. Maraschi, MNRAS 368, L52-L56 (2006), arXiv:0603030.

[14] J. Sitarek \& W. Bednarek, Ap\&SS 309, 105-109 (2007), arXiv : 0610313.

[15] F. A. Aharonian, D. Khangulyan \& L. Costamante, MNRAS 387, 1206-1214 (2008).

[16] D. Mazin \& M. Raue, Astronomy \& Astrophysics 471, 439-452 (2007), arXiv:astro-ph/0701694.

[17] M. R. Orr, F. Krennrich, and E. Dwek, The Astrophysical Journal, 733, 77 (2011).

[18] A. Dominguez, J. D. Finke, F. Prada, et al., The Astrophysical Journal, 770, 77 (2013).

[19] A. A. Abdo, M. Ackermann, M. Ajello, et al., The Astrophysical Journal, 723, 1082 (2010).

[20] M. Ackermann, M. Ajello, A. Allafort, et al., Science, 338, 1190-1192 (2012).

[21] A. Abramowski, F. Acero, F. Aharonian, et al., Astronomy \& Astrophysics, 550, A4 (2013).

[22] A. Franceschini, G. Rodighiero \& M. Vaccari, Astronomy \& Astrophysics 487, 837-852 (2008), arXiv:0805.1841.

[23] T. M. Kneiske \& H. Dole, Astronomy \& Astrophysics 515, 19 (2010)

[24] R. C. Gilmore, R. S. Somerville, J. R. Primack, and A. Dominguez MNRAS 422, 3189 (2012)

[25] B. S. Acharya, M. Actis, T. Aghajani, et al., Astropart. Phys. 43, 3-18 (2013)

[26] M. Raue \& D. Mazin, Astropart. Phys. 34, 245-256 (2010), arXiv: 1005.1196

[27] D. Mazin, M. Raue, B. Behera, et al., Astropart. Phys. 43, 241-251 (2013) 
[28] A. de Angelis, M. Roncadelli \& O. Mansutti, Phys. Rev. D 76, 121301 (2007), arXiv: 0707.4312.

[29] D. Horns \& M. Meyer, J. Cosmology Astropart. Phys. 2, 33 (2012)

[30] S. Archambault, T. Aune, B. Behera, et al., The Astrophysical Journal 785, 16 (2014)

[31] W. Essey \& A. Kusenko, Astropart. Phys. 33, 81 (2010)

[32] S. Razzaque, C. D. Dermer \& J. D. Finke, The Astrophysical Journal 745, 196 (2012)

[33] W. Essey \& A. Kusenko, Astropart. Phys. 57, 30 (2014)

[34] D. Horns, L. Maccione, M. Meyer, et al., Phys. Rev. D 86, 075024 (2012)

[35] M. Ackermann, M. Ajello, A. Allafort, et al., ApJS 209, 34 (2013) 